\title{
Variations on the Roy-Gallai theorem
}

\author{
Dominique de Werra $^{1}$ and Pierre Hansen ${ }^{2}$ \\ 1 Institut de Mathématiques, Ecole Polytechnique Fédérale de Lausanne, 1015 Lausanne, Switzerland \\ (e-mail: dewerra.ima@epfl.ch) \\ 2 GERAD and Département des Méthodes Quantitatives de Gestion, \\ Ecole des Hautes Etudes Commerciales, Montréal, Canada \\ (e-mail: pierreh@crt.umontreal.ca)
}

Received: December 2003 / Revised version: July 2004

\begin{abstract}
A generalization of the Roy-Gallai Theorem on the chromatic number of a graph is derived which is also an extension of several other results of Berge and of Li. A simple inductive proof is given which provides a direct way of deriving the Theorem of Li. We also show that some classical results valid for optimal colorings cannot be transposed to suboptimal colorings. We finally investigate some elementary properties which are also valid in suboptimal colorings.
\end{abstract}

Key words: Graph coloring, chromatic number, longest path, panchromatic chains, stable sets

AMS classification: 05C15, 05C38

\section{Introduction}

Bounds on the chromatic number of a graph have been obtained by using various coloring techniques like sequential coloring of nodes or construction of circuit-free orientations of edges.

In this note we exploit further the relation between orientations and colorings and we generalize the results in (Li 2001) and in (de Werra and Hansen 2003); in addition we provide a simple inductive proof of a Theorem of ( $\mathrm{Li} 2001$ ). From these results, the Roy-Gallai Theorem (Roy 1967; Gallai 1968) as well as some generalizations of (Berge 1982) can be derived. We also investigate the case of suboptimal colorings (i.e., colorings where the number of colors used is not minimum).

For all graph theoretical terms not defined here the reader is referred to (Berge 1973). 


\section{Preliminary results}

Recall that a graph $G=(V, E)$ is simple if it has neither multiple edges nor loops. $V$ (or $V(G)$ ) is a finite set of nodes and $E$ (or $E(G)$ ) is a family of edges [ $v, w]$ (unordered pairs). A graph $G=(V, U)$ is oriented if $U$ consists of a family of ordered pairs $(v, w)$, called arcs.

A set $S$ of nodes of $G$ is stable if no two nodes in $S$ are linked by an edge (or an arc).

A $k$-coloring of $G$ is a partition of its node set $V$ into $k$ stable sets $S_{1}, S_{2}, \ldots, S_{k}$; this amounts to assigning to each node $v$ a color $c(v)$ in $\{1, \ldots, k\}$ in such a way that no two adjacent nodes have the same color.

It is not difficult to see that a simple graph $G$ admits a $k$-coloring iff its edges can be oriented in such a way that the resulting graph $G^{\prime}$ has no (oriented) circuit and no path in $G^{\prime}$ has more than $k$ nodes.

Exploiting this connection between colorings and orientations, B. Roy and T. Gallai obtained independently the following result for oriented graphs where circuits may be present:

Theorem 1 (Roy 1967; Gallai 1968). If in an oriented graph $G$, every elementary path has at most $k$ nodes, then $G$ has a k-coloring.

Let us recall the definition of the chromatic number of $G$ denoted by $\chi(G)$ ): it is the smallest $k$ for which $G$ has a $k$-coloring. If $s(P)$ is the number of nodes on a path $P$, Theorem 1 can be stated as follows:

$$
\chi(G) \leq \max \{s(P) \mid P \text { is an elementary path in } G\} .
$$

In ( $\mathrm{Li} 2001)$ a direct extension of Theorem 1 was given by considering the root of a graph $G$; the root is a node $r$ such that for any other node $w$ of $G$ there is a path from $r$ to $w$. Calling $R(G)$ the set of roots of $G$ (which may be empty) and defining

$p(r)=\max \{s(P) \mid P$ is an elementary path in $G$ starting at node $r\}$, one can state:

Theorem 2 (Li 2001). For any oriented graph $G=(V, U)$

$$
\chi(G) \leq \min (|V|, \min (p(v) \mid v \in R(G))) .
$$

It is obvious that Theorem 2 implies Theorem 1 for all graphs such that $R(G) \neq \emptyset$. In the same paper, Li obtains another result which can be formulated as follows:

Theorem 3 (Li 2001). Let $G$ be a connected graph and let $k=\chi(G)$; then for any $k$-coloring of $G$ and for any node $v$ there exists an elementary chain starting at $v$ which meets all $k$ colors. 
However, as mentioned in (Li 2001), Theorem 3 cannot be applied to oriented graphs (a cycle $C_{5}$ on 5 nodes with an adequate orientation of its edges provides a counter-example).

In (de Werra and Hansen 2003) a generalization of Theorem 2 was derived by using the concept of basis of an oriented graph; it is a subset $B$ of nodes of $G$ such that:

a) for every node $w$ not in $B$, there is some node $b$ in $B$ from which an elementary path reaches $w$;

b) no two nodes in $B$ are linked by a path.

Observe that a basis $B$ is a stable set; unlike roots, bases always exist.

Let us define for a stable set $S$ the number $p(S)$ by

$$
p(S)=\max \{p(b): b \text { is a node of } S\} .
$$

Then the following result can be stated where a stable set is called basic if it contains some basis of $G$.

Theorem 4 (de Werra and Hansen 2003). For any oriented graph $G$

$$
\chi(G) \leq \min \{p(S) \mid S \text { is a basic stable set }\} .
$$

Clearly Theorem 4 implies Theorem 2 (and Theorem 1). It also implies a result of (Berge 1982) as shown in (de Werra and Hansen 2003), i.e., if $k$ is the largest number of nodes in a path of an oriented graph $G$ and if $P$ is a path with $k$ nodes, there is a $k$-coloring of $G$ such that $P$ meets every color (exactly once).

For the unoriented case, we may define $q(S)$ as the maximum number of nodes in an elementary chain starting at some node in $S$ and having no other node in $S$. Then one gets:

Corollary (de Werra and Hansen 2003). For any simple graph $G$

$$
\chi(G) \leq \min \{q(S) \mid S \text { is a stable set }\} .
$$

In the next section we shall derive a result which is a generalization of all unoriented versions of the above theorems.

For the moment let us recall a result given in (Gyarfas et al. 1980) and in (Sumner 1981):

Proposition 5. Let $G$ be a graph with $\chi(G)=k$ and $T=(W, F)$ a tree on $k$ nodes; let $f: V \rightarrow\{1, \ldots, k\}$ be a $k$-coloring of $G$ and $g: W \rightarrow\{1, \ldots, k\}$ an injection. Then there exists an edge preserving injection $h: W \rightarrow V$ such that $g(v)=f(h(v))$ for any $v \in W$. 
This implies in particular that in any $k$-coloring of $G$ (with $\chi(G)=k$ ) for any order $a_{1}, a_{r}, \ldots, a_{k}$ of the colors there exists a chain which meets each color exactly once in the order $a_{1}, \ldots, a_{k}$ (just take $T=$ chain on $k$ nodes $1,2, \ldots, k$; then $\left.g(i)=a_{i}\right)$. It also follows that in any $\chi(G)$-coloring of $G$ there exists a node $v$ such that $N[v]$ contains $\chi(G)$ colors (take $T=$ star formed by edges $\left[w_{1}, w_{i}\right]$ for $i=2, \ldots, \chi(G)$ and $g\left(w_{i}\right)=i$ for $\left.i=1, \ldots, \chi(G)\right)$.

At this stage one may wonder whether these properties carry over to the case where one has a $q$-coloring with $q>\chi(G)$; in other words, let $G$ be a graph and $\top(W, F)$ a tree on $k=\chi(G)$ nodes; let $f: V \rightarrow\{1, \ldots, q\}$ be a $q$-coloring (with $q>\chi(G))$ of $G$ and $g: W \rightarrow\{1, \ldots, q\}$ an injection. Does there exist an edge preserving injection $h: W \rightarrow V$ such that $g(v)=f(h(v))$ for any $v \in W$ ?

Rather unexpectedly, the answer is negative as can be seen: a graph $G$ (called penta $K_{3,3}$ ) has been constructed by I. Blöchliger which satisfies $\chi(G)=4$. A 5-coloring $f$ of $G$ can be constructed in such a way that for any node $v, N[v]$ contains at most 3 colors. This means that by taking a tree $T$ formed by edges $\left[w_{1}, w_{i}\right] i=2,3,4$ and $g\left(w_{i}\right)=i(1 \leq i \leq 4)$, one cannot find an edge preserving injection $h:\left\{w_{1}, w_{2}, w_{3}, w_{4}\right\} \rightarrow V$ such that $g(w)=f(h(w))$ for any $w \in W$.

The graph penta $K_{3,3}$ is constructed as follows (see Blöchliger and de Werra 2003): let $C=\{1, \ldots, 5\}$, then for every $c \in C$ and every subset $\{i, j\}$ of $C-\{c\}$, construct a node $(c, i, j)$. So we obtain 30 nodes $((c, i, j)$ and $(c, j, i)$ are the same node).

Now nodes $(c, i, j)$ and $(\bar{c}, \bar{i}, \bar{j})$ are linked by an edge iff $c \in\{\bar{i}, \bar{j}\}$ and $\bar{c} \in$ $\{i, j\}$. By giving color $c$ to all nodes $(c, i, j)$ we get a 5-coloring $f$ such that $N[v]$ contains 3 colors for each node $v$. One can furthermore show that $\chi(G)=4$ (see Blöchliger and de Werra 2003).

As a conclusion $q$-colorings (with $q>\chi(G)$ ) are less structured than $\chi(G)$ colorings as can be expected, and it follows that results like Proposition 5 cannot be directly transposed to that case.

In the remainder of the paper we shall concentrate on some properties which can be extended to $q$-colorings and which can be derived by simple techniques.

\section{Panchromatic chains}

Let us now state a direct extension of Theorem 3 which is similar to Theorem 4 and its corollary in the sense that it involves stable sets as well. Recall that a $k$-critical graph $G$ is such that $\chi(G)=k$, but removal of any node $v$ gives a graph $G^{\prime}=G-v$ with $\chi\left(G^{\prime}\right) \leq k-1$.

In a graph $G$ with $\chi(G)=k$ and with a $k$-coloring $\left(S_{1}, \ldots, S_{k}\right)$ a set $C$ of nodes (or a partial graph defined on $C$ ) will be called panchromatic if $C \cap S_{i} \neq \emptyset$ for $i=1, \ldots, k$. The next result deals with colorings which may also be suboptimal $(k \geq \chi(G))$. 
Theorem 6. Let $G$ be a connected graph and let $k$ be an integer with $k \geq \chi(G)$; let furthermore $H$ be a $\chi(G)$-critical subgraph of $G$ and $\left(S_{1}, S_{2}, \ldots, S_{k}\right)$ a k-coloring of $G$.

Then for any subset of nodes $S \subseteq S_{1}$, there is an elementary chain $C$ which starts at some node of $S$, has no other node in $S$ and meets at least $\chi(G)$ colors.

In addition after entering into $H$, chain $C$ remains inside $H$.

Corollary 7. Let $G$ be a connected graph with $\chi(G)=k$ and $\left(S_{1}, \ldots, S_{k}\right)$ a $k$ coloring of $G$. Then for any $S \subseteq S_{1}$, there is an elementary panchromatic chain $C$ starting in $S$ and such that $|C \cap S|=1$.

It is clear that Theorem 6 implies Theorem 3: take a subset $S$ with $|S|=1$ and $k=\chi(G)$; it also implies the Corollary of Theorem 4 since the chain $C$ is allowed to have only its starting node in the stable set $S$, in the same way as in the corollary.

Proof of Theorem 6. We shall give a proof by induction on the number $n$ of nodes and the chromatic number $\chi(G)$ of $G$. The result is trivial for a connected graph $G$ with 2 nodes and with $\chi(G)=2$.

Let us assume that the result is true for graphs with at most $n-1$ nodes and chromatic number at most $p-1$.

Let now $G=(V, E)$ be a graph with $|V|=n$ nodes and chromatic number $\chi(G)=p \leq k ;\left(S_{1}, \ldots, S_{k}\right)$ is a $k$-coloring of $G$. Consider now a $\chi(G)$-critical subgraph $H$ of $G$; notice that $\chi(H)=p$ and $H$ is connected.

Let $S \subseteq S_{1}$ and let $S^{H}=S \cap V(H)$. Let $V^{*}$ be a set of nodes such that the subgraph $H^{\prime}=H-\left(S_{1} \cup V^{*}\right)$ obtained by removing the nodes of $S_{1} \cup V^{*}$ from $H$, has $\chi\left(H^{\prime}\right)=p-1$. Then $H^{\prime}$ (which is possibly not connected) has some connected component, say $H^{\prime \prime}$, with $\chi\left(H^{\prime \prime}\right)=p-1$ (otherwise we would have $\left.\chi\left(H^{\prime}\right)<p-1\right)$. Since $S_{1} \neq \emptyset$, we have $\left|V\left(H^{\prime \prime}\right)\right| \leq\left|V\left(H^{\prime}\right)\right| \leq n-1$; so the theorem holds for $H^{\prime \prime}$ by the induction hypothesis, i.e., by choosing a subset $S=\left\{v^{\prime \prime}\right\}$ there exists from any node $v^{\prime \prime}$ in $V\left(H^{\prime \prime}\right)$ an elementary chain $C^{\prime \prime}$ which meets at least $p-1$ of the colors $2,3, \ldots, k$ and which remains inside $H^{\prime \prime}$ and hence inside $H$.

A) Assume now that $S^{H} \neq \varnothing$. Let us choose some node $v$ in $S^{H}$; since $H$ is connected there exists an elementary chain between node $v$ and any node in $H^{\prime \prime}$. Let us consider such a chain $\hat{C}$; we follow its edges from $v$ in $S^{H}$ and interrupt it as soon as we reach a node $v_{o}^{\prime \prime}$ in $H^{\prime \prime}$. All intermediate nodes of such a $\hat{C}$ are in $V(H)-V\left(H^{\prime \prime}\right)(\hat{C}$ remains in $H)$; now following the chain $\hat{C}$ in reverse order from $v_{o}^{\prime \prime}$ we follow its edges until we meet a first node in $S^{H}$; let $w$ be this node. Such a $w$ exists since $v$ is in $S^{H}$; we remove all edges of $\hat{C}$ between $v$ and $w$. The remaining chain $\bar{C}$ from $w$ to $v_{o}^{\prime \prime}$ can be extended by a chain from $v_{o}^{\prime \prime}$ in $H^{\prime \prime}$ which meets at least $p-1$ of the colors $2,3, \ldots, k$. The resulting chain $C$ meets at least $p$ colors (since its first node $w$ is in $S^{H} \subseteq S_{1}$ ); it remains inside $H$ and it is elementary. Finally $V(C) \cap S^{H}=\{w\}$. 
B Assume that $S^{H}=\emptyset$, i.e., $S \subseteq S_{1}-V(H)$. Since $G$ is connected there is an elementary chain from an arbitrary node $v$ in $S$ to some arbitrary node $u$ in $H$; we interrupt such a chain at the first node which is in $H$. As before we may destroy from the beginning of the chain all edges which are traversed before we leave $S$ for the last time. Let $C_{1}$ be the resulting chain from a node $\bar{v}$ in $S$ to a node $\bar{u}$ in $H$.

Now if $\bar{u}$ is in $V\left(H^{\prime \prime}\right)$, we are done: there is by the induction assumption an elementary chain $C_{2}$ in $H^{\prime \prime}$ from $\bar{u}$ which meets at least $p-1$ of the colors $2,3, \ldots, k$. Concatenation of $C_{1}$ and $C_{2}$ gives the required chain of $G$.

Finally assume $\bar{u}$ is in $V(H)-V\left(H^{\prime \prime}\right)$; now since $H$ is connected, there is an elementary chain (inside $H$ ) from $\bar{u}$ to some arbitrary node $v^{\prime \prime}$ in $V\left(H^{\prime \prime}\right)$; this chain from $\bar{u}$ can be interrupted at the first node $\bar{v}^{\prime \prime}$ which is in $V\left(H^{\prime \prime}\right)$; then, as before, it can be extended by a chain from $\bar{v}^{\prime \prime}$ which remains inside $H^{\prime \prime}$ and meets at least $p-1$ of the colors $2,3, \ldots, k$. Combining this chain $C_{2}$ with the chain $C_{1}$ from $\bar{v}$ to $\bar{u}$, we get the required chain $C$ in $G$; it satisfies $V(C) \cap S=\{u\}$ : since $S_{H}=\emptyset$, when the chain enters into $H$, it remains inside and it does not meet any node of $S$ other than $v$.

Remark 1. The above inductive proof (where $k=\chi(G), H$ is omitted and $S$ chosen with $|S|=1$ ) provides a simple way of proving Theorem 3 directly; the resulting derivation appears to be much simpler than the original proof of ( $\mathrm{Li} 2001)$.

Remark 2. The statement of Theorem 6 is best possible in the following sense: if $S$ is not monochromatic, the statement is not true:

consider a cycle $C_{7}$ on 7 nodes $a, b, c, d, e, f, g$; take $S_{1}=\{a, c, e\}, S_{2}=$ $\{b, d, f\}, S_{3}=\{g\}$ and $S=\{b, e, g\}$. Then there is no elementary chain out of $S$ which meets all colors and has only its first node in $S$.

In addition, one cannot choose arbitrarily the node $v$ in $S$ from which the chain $C$ is constructed: take the same coloring of the graph $C_{7}$ and $S=S_{1}$, then there is no chain out of node $c$ in $S$ which meets all colors.

Finally we mention another direct consequence of the above result:

Corollary 8 (de Werra and Hansen 2003). Let $G=(V, E)$ be a connected simple graph and let $k=\chi(G)$. Let $H$ be a $k$-critical subgraph of $G$. Then for any $k$ coloring of $G$ and for any subset $S \subseteq V$, there is a collection $C$ of $|S|$ node disjoint chains starting at nodes in $S$, having no other nodes in $S$ and meeting together all $k$ colors in $H$. Furthermore if achain of $C$ enters into $H$ it remains inside.

\section{Stars and flutes}

It is well known that the following statement holds:

Let $\left(S_{1}, \ldots, S_{k}\right)$ be any $k$-coloring of a graph $G$ with $\chi(G)=k \geq 2$. Then there is a connected subgraph $H$ of $G$ with diameter at most 2 which meets every color class exactly once. 
It is worth observing that it is not true if $k>\chi(G)$ : a chain $P_{4}$ on 4 nodes colored with $1,2,3,4$ is a counter example.

At this stage, we would like to mention a consequence of the equivalence between colorings and circuit-free orientations.

In a graph $G$ with $\chi(G) \leq k$, given a $k$-coloring $\left(S_{1}, \ldots, S_{k}\right)$, a flute $F$ will be a chain such that $\left|F \cap S_{i}\right| \leq 1$ for $i=1, \ldots, k$. So a panchromatic flute meets all $k$ colors exactly once. The size $|F|$ of a flute $F$ will be the number of its nodes.

Let us now consider a $k$-coloring of a graph $G$ with $k=\chi(G)$. We have the following well known consequence of Proposition 5:

for any $k$-coloring of $G$ with $\chi(G)=k$ and for any permutation $a_{1}, a_{2}, \ldots, a_{k}$ of the colors $1,2, \ldots, k$ there exists a panchromatic flute which meets colors $a_{1}, a_{2}, \ldots, a_{k}$ in this order.

This property does of course not hold when $k>\chi(G)$ : for a chain on three nodes colored with 1,2, 3 there is no flute $F$ with $|F|=2$ meeting consecutively colors 1 and 3 .

More generally, we can state

Proposition 9. Let $G$ be a graph and $\chi(G)$ its chromatic number. Let $k$ be an integer with $k \geq \chi(G)$. For any $k$-coloring of $G$ there is at least one flute $F$ with $|F| \geq \chi(G)$ meeting its colors in increasing order.

Proof. We construct the orientation associated to the $k$-coloring (edge $[u, v]$ becomes arc $(u, v)$ if the colors $c(u), c(v)$ of its end nodes satisfy $c(u)<c(v))$.

In the oriented graph $G$, there is no circuit and there is at least one path $P$ with at least $\chi(G)$ nodes; indeed if there would be no such paths, $G$ could be colored with less than $\chi(G)$ colors, a contradiction.

Now path $P$ is a flute which meets at least $\chi(G)$ colors in increasing order.

For claw free graphs we can give a more specific statement on the existence of a panchromatic flute.

A graph is claw-free if it does not contain a complete bipartite graph $K_{1,3}$ as an induced subgraph. $N[v]$ will again denote the set $\{v\} \cup N(v)$ where $N(v)$ is the set of neighbors of $v$.

Proposition 10. Let $G$ be a claw-free graph with chromatic number $\chi(G)$; then for any $k$-coloring $\left(S_{1}, \ldots, S_{k}\right)$ with $k \geq \chi(G)$ and for any node $v$, the set $N[v]$ contains a flute $G$ meeting all colors present in $N[v]$.

Proof. Consider a node $v$ and assume $\cup\left\{i \mid S_{i} \cap N(v) \neq \emptyset\right\}=\{1, \ldots, r\}$; let $v_{i}$ be a node of $N(v) \cap S_{i}$ for $i=1, \ldots, r$.

Claim. Let $Q$ be a connected component of $N(v)$; then there is a chain meeting all nodes of $Q$. 
Proof of the claim. The result is trivial if $Q$ has at most 3 nodes. Let $Q$ have at least 4 nodes and assume the result is not true. Let $R$ be a chain in $Q$ meeting the largest possible number of nodes of $Q$. There is at least one node, say $\bar{v}$, which is not met by $R$. Since $Q$ is connected, we may even assume that $\bar{v}$ is at distance 1 from $R$, i.e., there is an edge $[\bar{v}, u]$ where $u$ is in the chain. Observe that $u$ cannot be the first nor the last node of $R$ since otherwise $R \cup[\bar{v}, u]$ would form a chain meeting more nodes of $Q$ than $R$. So let $a$ and $b$ be the first and the last nodes of $R$; clearly $a$ and $\bar{v}$ (resp. $b$ and $\bar{v}$ ) are not linked. Hence $a$ and $b$ must be linked, otherwise $v, a, b, \bar{v}$ induce a claw in $G$. But then starting at $\bar{v}$ and traversing edge $[\bar{v}, u]$ and the subchain $R(u, b)$ of $R$ between $u$ and $b$, followed by $[b, a]$ and the subchain $R\left(a, u^{*}\right)$ of $R$ between $a$ and $u^{*}$ (the predecessor of $u$ in $R$ ), we get a chain meeting more nodes of $Q$ than $R$. This is not possible. Hence there exists in $Q$ a chain, which visits all nodes of $Q$. This ends the proof of the claim.

Now since $G$ is claw-free, the set $\left\{v_{1}, \ldots, v_{r}\right\} \subset N(v)$ has at most two connected components.

If there is one, say $Q_{1}$, there exists from the claim a chain $R_{0}$ meeting all nodes of $Q_{1}$; then $C$ is obtained from $R_{0}$ by going from its last node $b$ to node $v$ by following $[b, v]$.

If there are two components $Q_{1}, Q_{2}$, let $b_{1}$ (resp. $a_{2}$ ) be the last (resp. first) node of a chain $R_{1}$ (resp. $R_{2}$ ) meeting all nodes of $Q_{1}$ (resp. $Q_{2}$ ). Then $C$ is obtained by following consecutively $R_{1},\left[b_{1}, v\right],\left[v, a_{2}\right], R_{2}$.

By setting $k=\chi(G)$ in Proposition 10 and by observing that in any $k$-coloring of a graph $G$ there is a node $v$ such that $N[v]$ contains all $\chi(G)$ colors, we obtain:

Corollary 11. If $G$ is a claw-free graph with chromatic number $\chi(G)$ amd $\left(S_{1}, \ldots, S_{k}\right)$ a $k$-coloring with $k=\chi(G)$, then there exists a node $v$ such that $N[v]$ contains a panchromatic flute.

As a conclusion, we mention a conjecture that would generalize previous results.

Conjecture. Given a connected graph with a $k$-coloring $\left(S_{1}, \ldots, S_{k}\right)$ for some $k \geq \chi(G)$ and a node $v$ of $G$, there exists an elementary chain $C=\left(v_{0}, v_{1}, \ldots, v_{q}\right)$ such that $v_{0}=v$ and the last $\chi(G)$ nodes form a flute.

The conjecture is true for $\chi(G)=3$ as shown below.

Proposition 12. Let $G$ be a connected graph with $\chi(G)=3,\left(S_{1}, \ldots, S_{k}\right)$ a $k$ coloring for some $k \geq 3$ and $v$ a node of $G$. There exists an elementary chain starting at $v$ and such that the last three nodes have different colors.

Proof. $G$ contains a 3-critical subgraph $H$ (which is an odd cycle). Assume first $v \notin H$ then there exists an elementary chain $C$ from $v$ to some node $v^{\prime}$ of $H$ containing no other node of $H$.

If $v \in H$, we set $v=v^{\prime}$. If $H$ is a triangle, we are done since its nodes have 3 different colors and for any choice of $v^{\prime}$ we can extend the chain as required to meet all three colors. 
Let $H$ be an odd cycle with at least 5 nodes; then for any $k$-coloring with $k \geq 3$ of $H$ there are at least two triples (no necessarily disjoint) of consecutive nodes with different colors. A node $v^{\prime}$ of $H$ cannot be the middle point of two triples, so we will always be able to extend the chain around $H$ from $v^{\prime}$ and meet a triple of consecutive nodes with all different colors.

Acknowledgements. This note was written in part while the first author was invited at the Université de Paris I (Panthéon-Sorbonne). The support of the CERMSEM (Centre de Recherches de Mathématiques, Statistique et Economie Mathématique) is gratefully acknowledged. The first author expresses his gratitude to Marc Demange for comments on an early version of this note.

The research was completed during a visit of the first author to GERAD (Centre d'Etudes et de Recherches en Analyse des Décisions) at Montreal (Canada). The first author was also supported by NSERC Grant, OGP00 39682 during this visit.

\section{References}

Berge C (1973) Graphs and hypergraphs. North Holland, Amsterdam

Berge C (1982) Diperfect graphs. Combinatorica 2: 213-222

Gallai T (1968) On directed paths and circuits. In: Theory of Graphs Proceed. Colloq. Tihany (1966). Academic Press, New York, pp 115-118

Hansen P, de Werra D (2002) Connectivity, transitivity and chromaticity: the pioneering work of Bernard Roy in graph theory. In: Bouyssou D (ed) Aiding decisions with multiple criteria. Kluwer, Boston, pp 23-42

Li H (2001) A generalization of the Gallai-Roy Theorem. Graphs and Combinatorics 17: 681-685

Roy B (1967) Nombre chromatique et plus longs chemins d'un graphe. Revue d'Informatique et de Recherche Opérationnelle 5: 129-132

de Werra D, Hansen P (2003) Using stable sets to bound the chromatic number. Information Processing Letters 87: 127-131

Gyarfas A, Szemeredi E, Tuza Zs (1980) Induced subtrees in graphs of large chromatic number. Discrete Mathematics 30: 235-244

Sumner D (1981) Subtrees of a graph and the chromatic number. In: Chartrand G (ed) The theory and applications of graphs. (Kalamazoo, 1980). Wiley, New York, pp 557-576

Blöchliger I, de Werra D (2004) On some properties of suboptimal colorings of graphs. Networks 43: $103-108$ 